\title{
LA CONSULTA A EXPERTOS, UN IMPORTANTE RECURSO EN LA INVESTIGACIÓN
}

\section{CONSULTATION WITH EXPERTS, AN IMPORTANT RESOURCE IN RESEARCH}

\author{
SILVESTRE ORTIZ, Jose Refugio \\ Universidad de Sonora (UNISON) \\ Departamento de Ingeniería Civil y Minas (DICYM) \\ Profesor Investigador de tiempo completo \\ Boulevard Rosales S/N, Colonia Centro, Hermosillo, Sonora, C.P. 83000. México \\ Correo electrónico: irsilves@dicym.uson.mx \\ Teléfono: +52 6622592184 / +526621550348
}

Palabras Clave: Oficina; consulta a expertos; rentabilidad inmobiliaria; calidad urbana

Key words: Office; consult experts; real estate profitability; urban quality

\section{Resumen}

Como el nombre de la ponencia propuesta lo indica, el objetivo de la misma es hacer notar la importancia que puede tener la consulta a expertos en el ámbito de la investigación. Dicha metodología fue utilizada en la tesis doctoral "Rentabilidad de los Inmuebles de Oficinas en el Área Metropolitana de Barcelona" (2017), a fin de calificar los factores de calidad urbana, ambiental y de aglomeración, es decir, aquellos que definen el entorno urbano y ambiental, sus edificaciones, accesibilidad, jerarquía social y aglomeración, mismos que inciden en la rentabilidad de las oficinas en el Área Metropolitana de Barcelona, España. Los factores calificados como "muy importantes" son: accesibilidad a avenidas importantes, accesibilidad a estación del metro, accesibilidad a grandes infraestructuras de transporte, calidad del stock edificado, compacidad económica-empresarial e intensidad del stock edificado. Los factores calificados como "importantes" son: accesibilidad a parada de autobús, accesibilidad a los clientes, calidad de urbanización, estado de conservación del stock edificado y nivel de ingresos.

\begin{abstract}
As the name of the proposed paper indicates, the objective of the same is to note the importance that can have the consultation to experts in the field of research. This methodology was used in the doctoral thesis "Profitability of office buildings at the Barcelona Metropolitan Area" (2017), in order to qualify, urban, environmental and agglomeration quality factors, that is, those that define the urban and environmental environment, its buildings, accessibility, social hierarchy and agglomeration, which affect the profitability of offices in the Metropolitan Area of Barcelona, Spain. Factors classified as "very important" are: accessibility to important avenues, accessibility to the metro station, accessibility to large transport infrastructures, quality of the built-up stock, economic-business compactness and intensity of the built-up stock. Factors classified as "important" are: accessibility to bus stop, accessibility to customers, quality of urbanization, state of conservation of built-up stock and level of income.
\end{abstract}




\section{Introducción}

En los diversos campos del saber, además del conocimiento construido mediante las investigaciones, existe un importante patrimonio intelectual en cada uno de los investigadores.

"La utilización del bagaje teórico práctico de los especialistas se convierte en un requerimiento para el desarrollo de cada campo del saber y en ocasiones, en una necesidad para el investigador que precisa apoyarse en la experticia de los colegas" (García et al, 2013).

En múltiples estudios se realizan consultas a sujetos que participan en calidad de "expertos". No obstante, convertir los conocimientos que poseen los especialistas en información científica, requiere de la aplicación controlada de un método.

La obtención de datos aportados por conocedores se realiza a través de varios métodos, en el caso de la consulta a expertos son comunes los grupos focales (valoraciones construidas en el consenso grupal directo) y las entrevistas (apreciaciones individuales). También se tienen los métodos de consenso que se ocupan esencialmente de obtener estimaciones estadísticas a partir de aproximaciones cualitativas. Dentro de éste tipo de consulta, destaca el Método Delphi como un caso especial.

En el presente trabajo se ha elegido como instrumento a la entrevista a expertos, a fin de estimar los factores urbanos y ambientales en el Área Metropolitana de Barcelona, que inciden en la rentabilidad de las oficinas; porque se considera lo adecuado al caso, la bondad y fortaleza de dicho método y los muy limitados recursos de que se dispone.

\section{Conceptos básicos, criterios y selección de expertos}

Por experto se entiende "aquel cuya formación y experiencia previa le ha permitido alcanzar un dominio sobre un asunto que excede el nivel promedio de sus iguales, y que está en disposición de exponer sus opiniones sobre dicho asunto para que sean utilizadas como juicios conclusivos. Se le considera apto para emitir criterios certeros por quien se los solicita" (García et al, 2013).

La selección de los expertos es una parte muy importante del método, Skjong y Wentworth (2000) proponen los siguientes criterios de selección: a) Experiencia en la realización de juicios y toma de decisiones basada en evidencia o experticia (grados, investigaciones, publicaciones, posición, experiencia, premios, entre otras), b) reputación en la comunidad, c) disponibilidad y motivación para participar y d) imparcialidad y cualidades inherentes como confianza en sí mismo y adaptabilidad.

También plantean que los expertos pueden estar relacionados por educación similar, entrenamiento, experiencia, entre otros. Mc Gartland, Berg, Tebb, Lee y Rauch (2003) proponen como criterio básico de selección únicamente el número de publicaciones o la experiencia. 
Para ésta investigación se han tomado los siguientes criterios de inclusión:

- El experto puede ser un Valorador Profesional con amplia experiencia y con conocimiento del Área Metropolitana de Barcelona. Si tiene relación con la docencia universitaria, es mejor.

- El experto puede ser un Operador Inmobiliario, con amplia experiencia en el mercado de oficinas y con conocimiento del Área Metropolitana de Barcelona.

En relación al número de expertos a utilizar, se dice que depende del nivel de experticia y de la diversidad del conocimiento, sin embargo, la decisión de cuántos expertos es adecuado, varía entre autores, Mc Gartland et al (2003) establecen un rango entre 2 y 20 expertos, Hyrkas et al (2003) proponen diez expertos para tener mayor confianza en la estimación.

Para éste trabajo, que consta de dos etapas, fue posible tener seis expertos para la primera etapa y tres para la segunda; también hay que hacer notar que se invitó a muchos más, algunos de ellos no contestaron, otros si lo hicieron, para disculparse porque sus actividades no permitían su colaboración.

Los perfiles de los seis expertos seleccionados en la etapa uno, son los siguientes:

\begin{tabular}{|c|c|}
\hline Exp. 1 & $\begin{array}{l}\text { Arquitecto especializado en urbanismo y valoraciones inmobiliarias. Profesor de } \\
\text { valoraciones inmobiliarias en la Escuela Técnica Superior de Arquitectura de } \\
\text { Barcelona, en la Universidad Politécnica de Cataluña. }\end{array}$ \\
\hline Exp. 2 & $\begin{array}{l}\text { Arquitecto valuador con reconocimiento europeo (REV), Arquitecto Técnico en } \\
\text { Ejecución de Obras, Ingeniero de Edificación, Técnico Superior en Prevención de } \\
\text { Riesgos Laborales y Máster Universitario en Valoraciones Inmobiliarias } \\
\text { Tasaciones y Peritaje Judicial. }\end{array}$ \\
\hline Exp. 3 & $\begin{array}{l}\text { Dr. Arquitecto, Valuador con reconocimiento europeo (REV), Máster en Asesoría } \\
\text { Inmobiliaria. Investigador titular en la Universidad Politécnica de Cataluña con } \\
\text { amplio conocimiento en la localización de oficinas en la Región Metropolitana de } \\
\text { Barcelona. }\end{array}$ \\
\hline Exp. 4 & $\begin{array}{l}\text { Profesor Titular de la Universidad de Sonora, en México. Desarrolló la tesis } \\
\text { doctoral "Influencia de los Indicadores de Calidad de Zona en la Rentabilidad y } \\
\text { Riesgo Inmobiliario: Caso Residencial Barcelona". }\end{array}$ \\
\hline Exp. 5 & Operador Inmobiliario de oficinas de la empresa INBISA en Barcelona, España. \\
\hline Exp. 6 & liario de Habitaclia en Barcelona, E \\
\hline
\end{tabular}

\section{Método a seguir en la consulta a expertos}

El procedimiento a seguir se presenta en la Figura 1, el proceso contiene cuatro etapas, la primera es la de preparación, en ella se plantea primeramente el objetivo a lograr con la consulta, se establecen criterios de selección para los expertos, se define el número de expertos deseable, se realizan las invitaciones a participar, se preparan las preguntas y se

Citación: SILVESTRE ORTIZ, J. R. La consulta a expertos, un importante recurso en la investigación. En: Libro de proceedings, CTV 2018. XII Congreso Internacional Ciudad y Territorio Virtual. "Ciudades y Territorios Inteligentes". UNCuyo, Mendoza, 5-7 septiembre 2018. Barcelona: CPSV, 2018, p. 181-192. 
define su escala de medición, en ésta parte, se recomienda realizar pruebas piloto para verificar que el instrumento sea adecuado y se planifica la forma de realizar las entrevistas.

\section{Figura 1. Método a seguir en la entrevista a expertos}

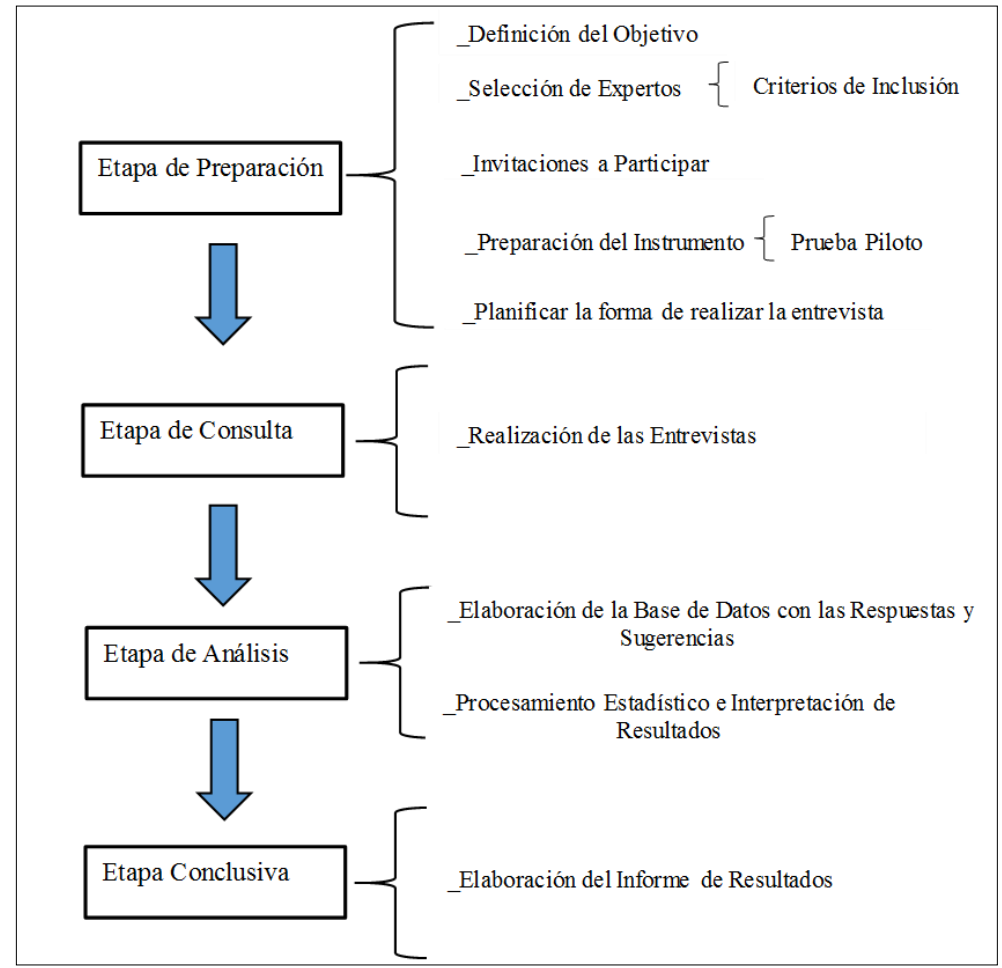

Fuente: Elaboración propia

En la etapa de consulta se realizan las entrevistas de la manera que se haya planificado, registrando todos los incidentes. En la etapa de análisis se construye la base de datos con las respuestas y sugerencias de los expertos, se realiza el proceso estadístico y se interpretan los resultados obtenidos. La etapa conclusiva consiste en la elaboración del reporte final de resultados.

Aplicando dicho procedimiento a nuestro caso, en secciones anteriores se especifica el objetivo de las entrevistas a expertos, así mismo se proporcionan los criterios de inclusión y los expertos seleccionados. En las secciones siguientes se continúa con el proceso para la etapa uno.

\section{Metodología de desarrollo}

\subsection{Cuestionario a los expertos y forma de aplicación (etapa uno)}

La entrevista se realiza individualmente y la comunicación es "cara a cara", el investigador lee las indicaciones del cuestionario y el experto evalúa y contesta cada ítem, el investigador llena el cuestionario según indique el experto. En la entrevista primeramente se establece el objetivo de la consulta, y se pide al experto evaluar cada factor en una escala ordinal del 1 al 4 , en donde 1 significa "sin importancia", el 2 "poco importante", el 3 "importante" y el 4 "muy importante".

Citación: SILVESTRE ORTIZ, J. R. La consulta a expertos, un importante recurso en la investigación. En: Libro de proceedings, CTV 2018. XII Congreso Internacional Ciudad y Territorio Virtual. "Ciudades y Territorios Inteligentes". UNCuyo, Mendoza, 5-7 septiembre 2018. Barcelona: CPSV, 2018, p. 181-192. 
El cuestionario a utilizar, se divide en cuatro aspectos, el primero consiste en factores urbanos de accesibilidad, el segundo se refiere a externalidades urbanas y ambientales, el tercero a jerarquía social y el cuarto a factores de aglomeración. Debe hacerse notar que al final de cada etapa, se pide al experto diga si hay sugerencias adicionales. También es importante mencionar que se realizaron dos pruebas pilotos para estar más seguros de lo adecuado de las preguntas. En las figuras siguientes (2 a 5) se presenta el cuestionario aplicado.

Figura 2. Entrevista a expertos, factores de accesibilidad

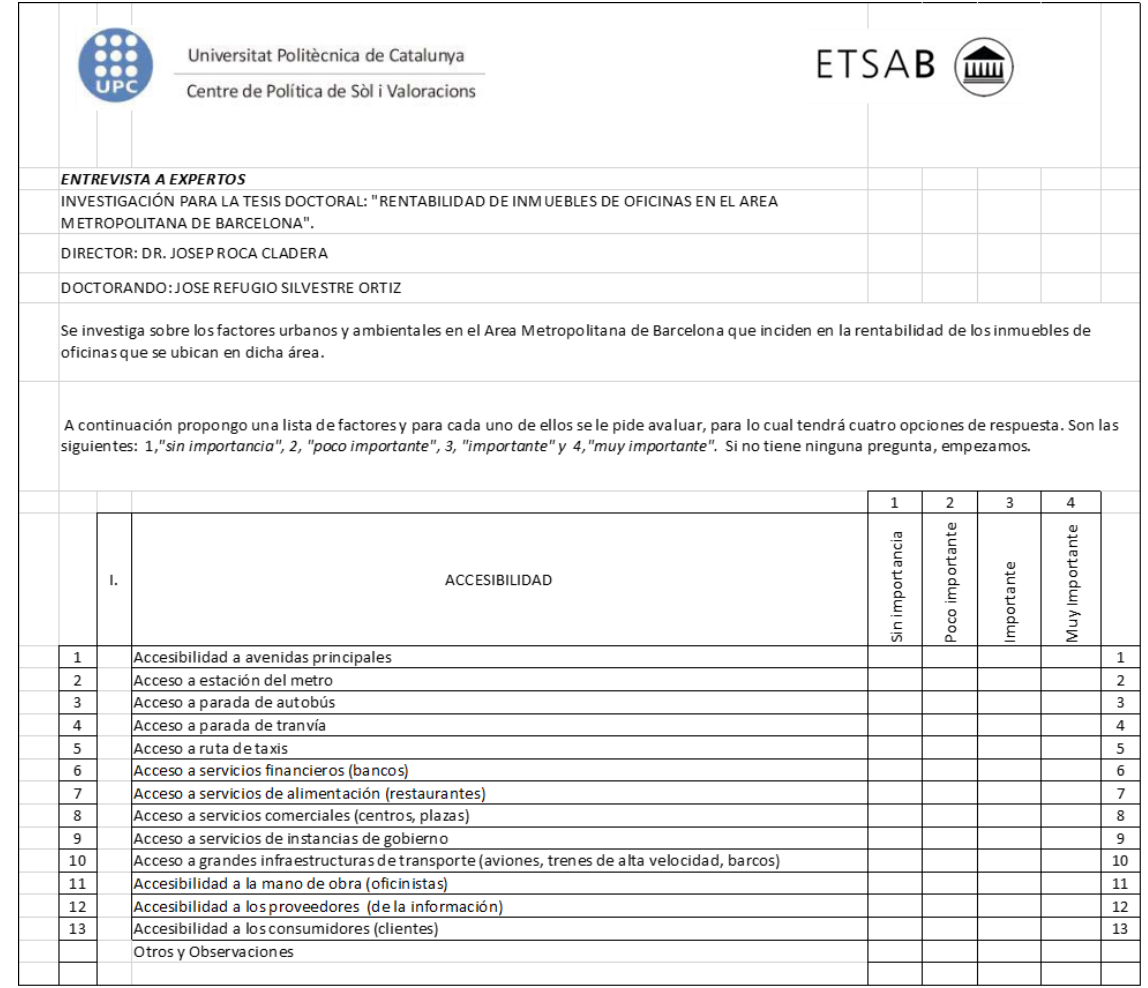

Fuente: Elaboración propia

Figura 3. Entrevista a expertos, factores de externalidades urbanas y ambientales

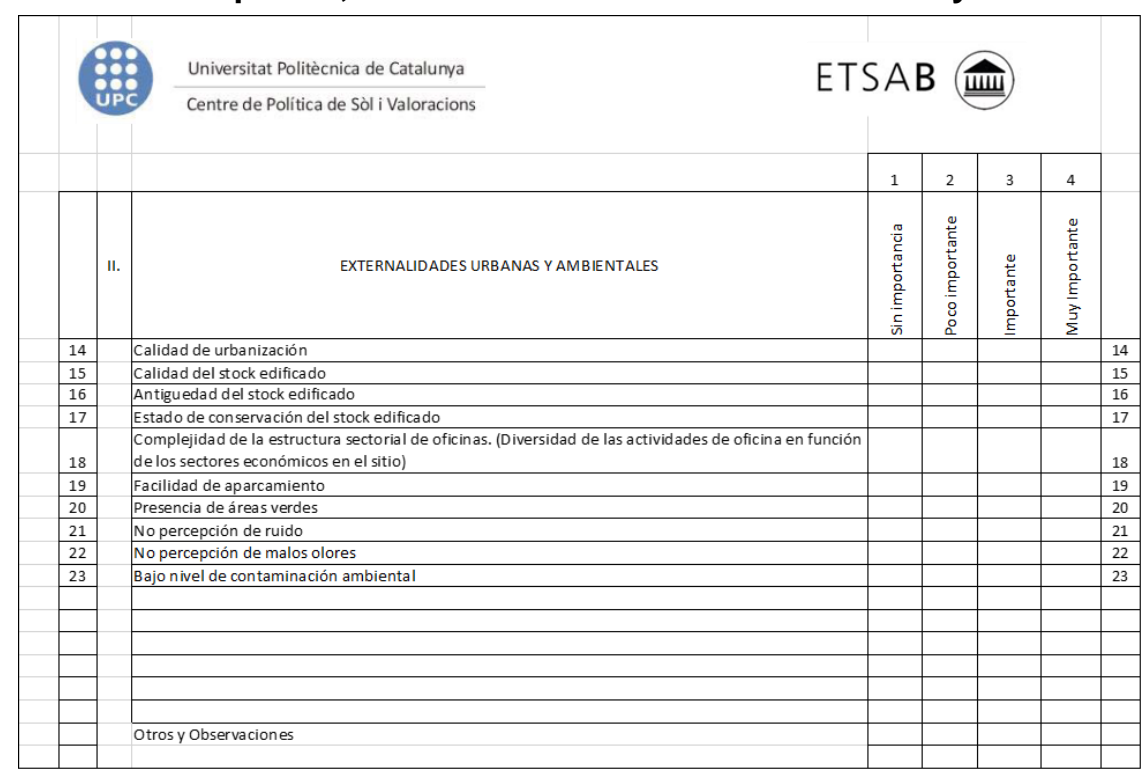

Fuente: Elaboración propia

Citación: SILVESTRE ORTIZ, J. R. La consulta a expertos, un importante recurso en la investigación. En: Libro de proceedings, CTV 2018. XII Congreso Internacional Ciudad y Territorio Virtual. "Ciudades y Territorios Inteligentes". UNCuyo, Mendoza, 5-7 septiembre 2018. Barcelona: CPSV, 2018, p. 181-192. 
Figura 4. Entrevista a expertos, factores de jerarquía social

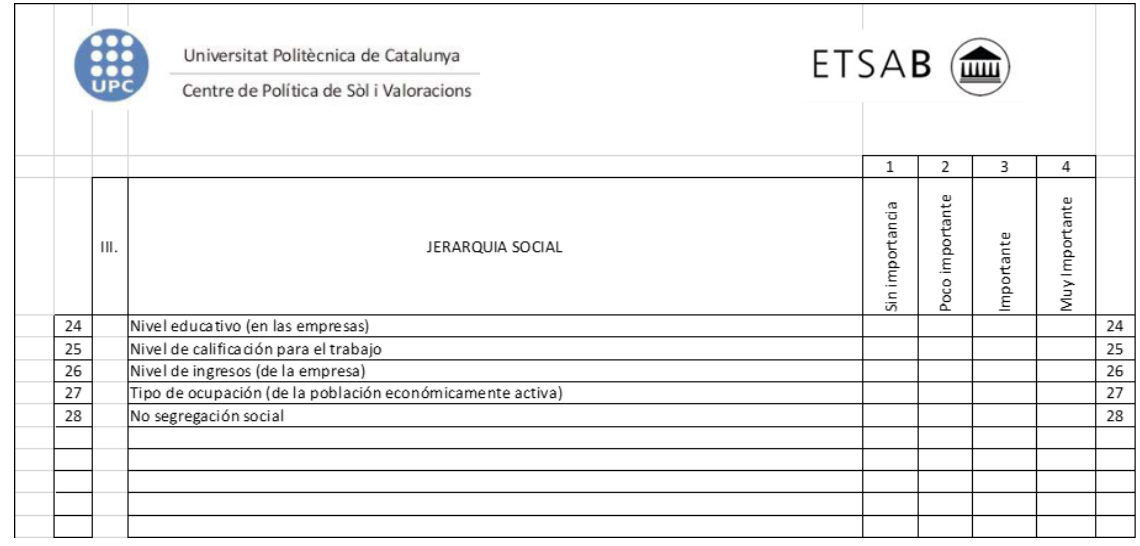

Fuente: Elaboración propia

Figura 5. Entrevista a expertos, factores de aglomeración

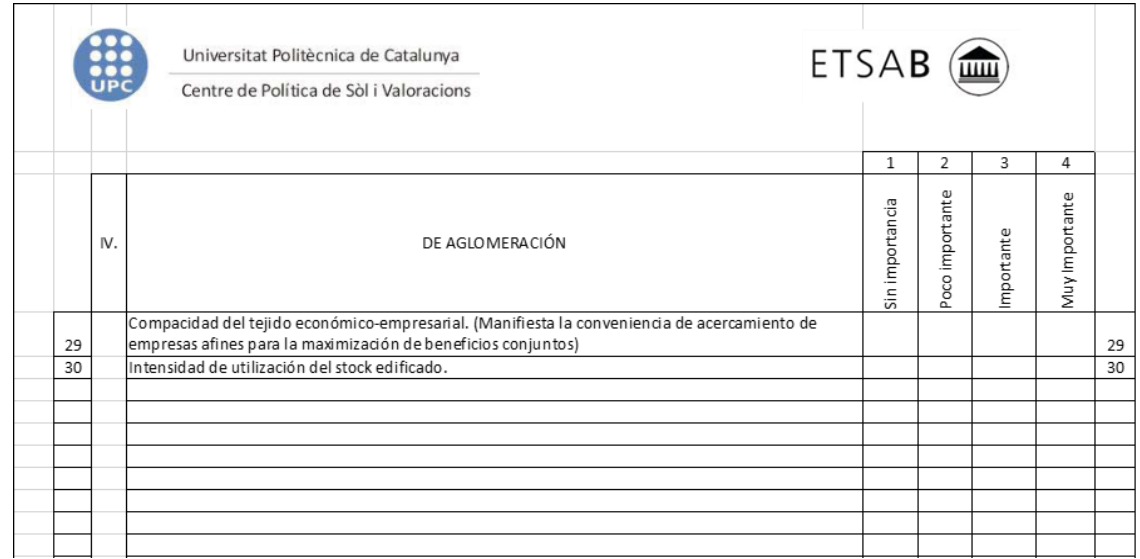

Fuente: Elaboración propia

\subsection{Realización de las entrevistas (etapa uno)}

El proceso se desarrolló sin mayores incidentes, salvo que en algún caso la entrevista se tuvo que reprogramar debido a que el experto tenía muchos compromisos.

\subsection{Respuestas de los expertos (etapa uno)}

Las tablas de la 1 a la 4, muestran las respuestas de los expertos al cuestionario anteriormente descrito, en la primera fila aparecen los factores ${ }^{1}$ en el mismo orden que se presentan en dicho

1 Dónde: acc_av_ppales, significa, accesibilidad a avenidas principales; acc_metro, significa, accesibilidad a estación
de metro; acc_bus, significa, accesibilidad a parada de autobús; acc_tram, significa, accesibilidad a estación de tranvía;
acc_taxis, significa, accesibilidad a rutas de taxis; acc_serv_fin, significa, accesibilidad a servicios financieros;
acc_serv_al, significa, accesibilidad a servicios de alimentación; acc_serv_com, significa, accesibilidad a servicios
comerciales; acc_serv_gob, significa, accesibilidad a servicios de gobierno; a_gdes_inf, significa, accesibilidad a
grandes infraestructuras de transporte; acc_m_de_o, significa, accesibilidad a la mano de obra; acc_prov_in, significa,
accesibilidad a los proveedores de la información; acc_consum, significa, accesibilidad a los consumidores (clientes);
cal_urb, significa, calidad de urbanización; cal_stk_ed, significa, calidad del stock edificado; ant_stk_ed, significa,
antigüedad del stock edificado; edo_cons_st, significa, estado de conservación del stock edificado; com_est_of,
significa, complejidad de la estructura sectorial de oficinas; fac_aparcto, significa, facilidad de aparcamiento;
áreas_vdes, significa, presencia de áreas verdes; no_ruido, significa, presencia de no ruido; no_m_olor, significa, no
presencia de malos olores; bja_cont_am, significa, presencia de baja contaminación ambiental; niv_educ, significa,
nivel educativo; calif_trab, significa, nivel de calificación para el trabajo; niv_ingresos, significa, nivel de ingresos;
tipo_ocupa, significa, tipo de ocupación; no_segr_soc, significa, no segregación social; comp_e_em, significa,
compacidad del tejido económico-empresarial; int_stk_ed, significa, intensidad de utilización del stock edificado

Citación: SILVESTRE ORTIZ, J. R. La consulta a expertos, un importante recurso en la investigación. En: Libro de proceedings, CTV 2018. XII Congreso Internacional Ciudad y Territorio Virtual. "Ciudades y Territorios Inteligentes". UNCuyo, Mendoza, 5-7 septiembre 2018. Barcelona: CPSV, 2018, p. 181-192. 
cuestionario, las siguientes seis filas son las respuestas y en la última fila con sombra, aparece el valor de la media aritmética para cada factor.

Tabla 1. Respuestas de entrevista a expertos, factores de accesibilidad

\begin{tabular}{|c|c|c|c|c|c|c|c|c|c|c|c|c|c|}
\hline acc_av_ppales & acc_metro & acc_bus & acc_tram & acc_taxis & acc_serv_fin & acc_serv_al & acc_serv_com & acc_serv_gob & a_gdes_inf & acc_m_de_o acc_prov_in & acc_consum \\
\hline 4 & 4 & 3 & 3 & 3 & 4 & 3 & 3 & 3 & 4 & 2 & 2 & 4 \\
\hline 4 & 3 & 3 & 3 & 2 & 2 & 2 & 2 & 2 & 3 & 2 & 2 & 3 \\
\hline 2 & 4 & 3 & 2 & 3 & 2 & 4 & 2 & 1 & 3 & 2 \\
\hline 3 & 3 & 3 & 2 & 2 & 3 & 2 & 2 & 2 & 3 & 3 & 3 \\
\hline 3 & 4 & 4 & 4 & 3 & 2 & 2 & 2 & 2 & 4 & 3 & 2 \\
\hline 4 & 3 & 2 & 3 & 1 & 2 & 2 & 1 & 2 & 3 & 3 & 3 & 4 \\
\hline 3,33 & 3,50 & 3,00 & 2,83 & 2,33 & 2,50 & 2,50 & 2,00 & 2,00 & 3,33 & 2,50 & 2,50 & 3,00 \\
\hline
\end{tabular}

Fuente: Elaboración propia

Tabla 2. Respuestas de entrevista a expertos, factores de externalidades urbanísticas y ambientales

\begin{tabular}{|c|c|c|c|c|c|c|c|c|c|}
\hline cal_urb & cal-stk_ed & ant_stk_ed & edo_cons_s & t comp_est_of & fac_aparcto & areas_vdes & no_ruido & no_m_olor & bja_cont_am \\
\hline 4 & 4 & 2 & 3 & 3 & 3 & 3 & 3 & 3 & 3 \\
\hline 3 & 4 & 3 & 3 & 1 & 3 & 2 & 2 & 2 & 2 \\
\hline 3 & 3 & 4 & 3 & 3 & 3 & 2 & 3 & 4 & 3 \\
\hline 3 & 3 & 3 & 3 & 2 & 3 & 2 & 2 & 2 & 2 \\
\hline 1 & 3 & 3 & 3 & 1 & 1 & 2 & 1 & 3 & 3 \\
\hline 4 & 4 & 2 & 3 & 3 & 2 & 1 & 2 & 2 & 2 \\
\hline 3,00 & 3,50 & 2,83 & 3,00 & 2,17 & 2,50 & 2,00 & 2,17 & 2,67 & 2,50 \\
\hline
\end{tabular}

Fuente: Elaboración propia

Tabla 3. Respuestas de entrevista a expertos, factores de jerarquía social

\begin{tabular}{|c|c|c|c|c|}
\hline niv_educ & calif_trab & niv_ingresos & tipo_ocupa & no_segr_soc \\
\hline 3 & 4 & 4 & 3 & 3 \\
\hline 3 & 1 & 4 & 3 & 1 \\
\hline 3 & 2 & 3 & 2 & 2 \\
\hline 2 & 3 & 3 & 2 & 2 \\
\hline 1 & 1 & 1 & 1 & 1 \\
\hline 3 & 3 & 3 & 3 & 1 \\
\hline 2,50 & 2,33 & 3,00 & 2,33 & 1,67 \\
\hline
\end{tabular}

Fuente: Elaboración propia

Tabla 4. Respuestas de entrevista a expertos, factores de aglomeración

\begin{tabular}{|c|c|}
\hline comp_e_em & int_stk_ed \\
\hline 4 & 4 \\
\hline 3 & 2 \\
\hline 2 & 3 \\
\hline 4 & 3 \\
\hline 4 & 4 \\
\hline 4 & 4 \\
\hline 3,50 & 3,33 \\
\hline
\end{tabular}

Fuente: Elaboración propia

\subsection{Análisis de resultados (etapa uno)}

Para factores calificados como IMPORTANTES se toma la siguiente regla de decisión: Seleccionar aquellos en donde la media aritmética de las puntuaciones sea 3.

La Tabla 5 muestra los resultados obtenidos. Destacando que el factor estado de conservación del stock edificado se califica como importante, siendo el único caso evaluado con el $100 \%$ de coincidencia de los expertos. 
Tabla 5. Factores evaluados por los expertos como Importantes

\begin{tabular}{|c|c|c|c|}
\hline Tipo de factor & Factor & $\bar{X}$ & Comentarios \\
\hline Accesibilidad & Acceso a parada de autobus & 3 & $67 \%$ de los expertos lo califican importante \\
\hline Accesibilidad & Acceso a los consumidores & 3 & $50 \%$ de los expertos lo califican importante \\
\hline Externalidades & Calidad de urbanización & 3 & $50 \%$ de los expertos lo califican importante \\
\hline Externalidades & $\begin{array}{c}\text { Estado de conservacion del } \\
\text { stock edificado }\end{array}$ & 3 & $100 \%$ de los expertos lo califican importante \\
\hline Jerarquia Social & Nivel de ingresos & 3 & $\begin{array}{c}50 \% \text { de los expertos lo califican importante y } \\
33 \% \text { como muy importante }\end{array}$ \\
\hline
\end{tabular}

Fuente: Elaboración propia

Para factores calificados como MUY IMPORTANTES se toma la siguiente regla de decisión: Seleccionar aquellos en donde la media aritmética de las puntuaciones sea superior a 3.

La Tabla 6 muestra los resultados obtenidos para factores MUY IMPORTANTES. Destacando la ausencia de factores de jerarquía social.

Tabla 6. Factores evaluados por los expertos como Muy Importantes

\begin{tabular}{|c|c|r|c|}
\hline Tipo de factor & Factor & $\bar{X}$ & Comentarios \\
\hline Accesibilidad & Acceso a avenidas principales & 3,33 & $\begin{array}{c}50 \% \text { de los expertos lo califican de muy } \\
\text { importante }\end{array}$ \\
\hline Accesibilidad & Acceso a estación del metro & 3,5 & $\begin{array}{c}50 \% \text { de los expertos lo califican de muy } \\
\text { importante y el otro } 50 \% \text { importante }\end{array}$ \\
\hline Accesibilidad & $\begin{array}{c}\text { Acceso a grandes } \\
\text { infraestructuras del transporte }\end{array}$ & 3,33 & $\begin{array}{c}67 \% \text { de los expertos lo califican de } \\
\text { importante y el 33\% de muy importante }\end{array}$ \\
\hline Externalidades & $\begin{array}{c}\text { Calidad del stock edificado } \\
\text { De aglomeración }\end{array}$ & 3,5 & $\begin{array}{c}\text { Compacidad economico de los expertos lo califican de muy } \\
\text { importante, el otro 50\% como importante }\end{array}$ \\
\hline De aglomeración & $\begin{array}{c}\text { Intensidad de utilización del } \\
\text { stock edificado }\end{array}$ & 3,5 & $\begin{array}{c}67 \% \text { de los expertos lo califican de muy } \\
\text { importante }\end{array}$ \\
\hline
\end{tabular}

Fuente: Elaboración propia

\subsection{Prueba estadística de concordancia W de Kendall}

La prueba contrasta una hipótesis nula que establece "los rangos son independientes y no hay concordancia", contra la alternativa que establece "hay concordancia significativa entre los rangos", basada en la distribución de probabilidad chi cuadrada, y un nivel de significación del $5 \%$, además el coeficiente $\mathrm{W}$ de Kendall proporciona el grado de concordancia (eliminando la influencia debida al azar) variando de 0 a 1, en donde 1 sería la concordancia perfecta. Dicha prueba se realiza con el software SPSS.

El test es útil para nuestro caso de consulta a expertos, dado que las variables son medidas ordinalmente y en una escala del 1 al 4 . La Tabla 4.7 muestra los resultados obtenidos. 
Tabla 7. Resultados de la prueba W de Kendall (Test Statistics)

a. Kendall's Coefficient of Concordance

\begin{tabular}{|l|r|}
\hline & 6 \\
Kendall's Wa &, 391 \\
Chi-Square & 67,999 \\
df & 29 \\
Asymp. Sig. &, 000 \\
\hline
\end{tabular}

Fuente: Elaboración propia

Como el valor de significación es 0 , inferior a 0.05 se rechaza la hipótesis nula y se concluye que hay concordancia significativa entre los rangos asignados por los expertos, siendo la fuerza de la concordancia de 0,391 . Por tanto, la prueba de Kendall establece que si hay concordancia entre los expertos, sin embargo el nivel de concordancia no es muy alto.

\subsection{Segunda etapa de consulta a expertos}

El objetivo de la segunda etapa es evaluar los factores obtenidos como "importantes" y "muy importantes" en la primera etapa de la consulta a expertos, relacionándolos con zonas de valor inmobiliarias.

\subsubsection{Cuestionario y forma de aplicación (etapa 2)}

De los seis expertos de la etapa uno ahora se selecciona únicamente a tres para ésta segunda etapa. La entrevista se realiza individualmente y la comunicación es "cara a cara", el investigador lee las indicaciones del cuestionario y el experto evalúa y contesta cada ítem, el investigador llena el cuestionario según indique el experto. En la entrevista primeramente se establece el objetivo de la consulta, y se pide al experto evaluar cada factor en una escala ordinal del 0 al 10, en donde 0 significa "no importante" y el 10 "de máxima importancia". En la Figura 6 se presenta el cuestionario aplicado.

Figura 6. Cuestionario a los expertos, en segunda etapa

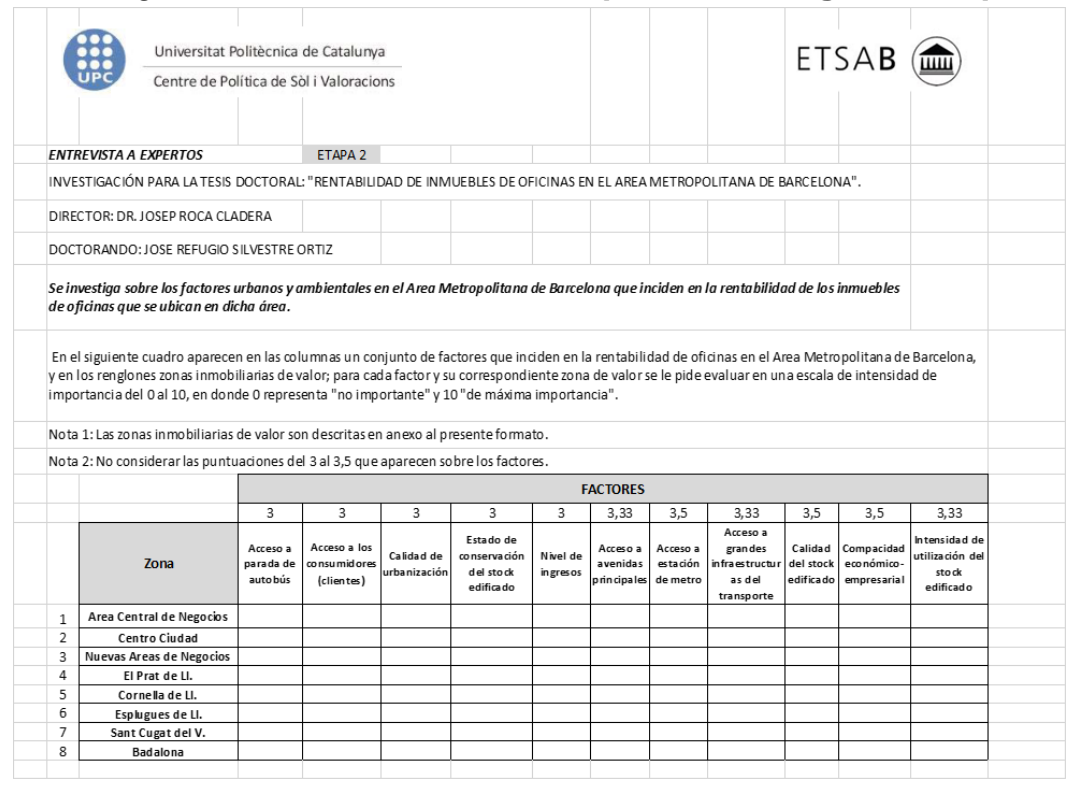

Fuente: Elaboración propia

Citación: SILVESTRE ORTIZ, J. R. La consulta a expertos, un importante recurso en la investigación. En: Libro de proceedings, CTV 2018. XII Congreso Internacional Ciudad y Territorio Virtual. "Ciudades y Territorios Inteligentes". UNCuyo, Mendoza, 5-7 septiembre 2018. Barcelona: CPSV, 2018, p. 181-192. 


\subsubsection{Realización de las entrevistas a los expertos (etapa 2)}

El proceso se desarrolló sin mayores incidentes.

\subsubsection{Análisis de resultados (etapa 2)}

La tabla 8, muestra la media aritmética de las puntuaciones obtenidas de los expertos.

Tabla 8. Media aritmética de las evaluaciones de expertos (etapa dos)

\begin{tabular}{|c|c|c|c|c|c|c|c|c|c|c|c|}
\hline & \multicolumn{11}{|c|}{ FACTORES } \\
\hline & 3 & 3 & 3 & 3 & 3 & 3,33 & 3,5 & 3,33 & 3,5 & 3,5 & 3,33 \\
\hline Zona & $\begin{array}{c}\text { Acceso a } \\
\text { parada de } \\
\text { autobús }\end{array}$ & $\begin{array}{c}\text { Acceso a los } \\
\text { consumidores } \\
\text { (clientes) }\end{array}$ & $\begin{array}{c}\text { Calidad de } \\
\text { urbanización }\end{array}$ & $\begin{array}{c}\text { Estado de } \\
\text { conservación } \\
\text { del stock } \\
\text { edificado }\end{array}$ & $\begin{array}{l}\text { Nivel de } \\
\text { ingresos }\end{array}$ & $\begin{array}{c}\text { Acceso a } \\
\text { avenidas } \\
\text { principales }\end{array}$ & $\begin{array}{c}\text { Acceso a } \\
\text { estación } \\
\text { de metro }\end{array}$ & $\begin{array}{c}\text { Acceso a } \\
\text { grandes } \\
\text { infraestructur } \\
\text { as del } \\
\text { transporte }\end{array}$ & \begin{tabular}{|c|} 
Calidad \\
del stock \\
edificado
\end{tabular} & $\begin{array}{l}\text { Compacidad } \\
\text { económico- } \\
\text { empresarial }\end{array}$ & $\begin{array}{c}\text { Intensidad de } \\
\text { utilización del } \\
\text { stock } \\
\text { edificado }\end{array}$ \\
\hline Area Central de Negocios & 5,33 & 9,67 & 9,33 & 8,67 & 9,00 & 9,33 & 8,67 & 5,67 & 8,67 & 9,67 & 10,00 \\
\hline Centro Ciudad & 5,67 & 9,33 & 8,67 & 8,00 & 8,33 & 8,67 & 9,00 & 6,33 & 8,00 & 9,33 & 9,00 \\
\hline Nuevas Areas de Negocios & 5,00 & 7,33 & 9,00 & 9,00 & 8,33 & 8,33 & 8,33 & 5,67 & 8,67 & 9,00 & 8,33 \\
\hline El Prat de Ll. & 6,00 & 5,67 & 8,33 & 8,33 & 6,00 & 6,33 & 6,33 & 8,00 & 8,33 & 7,00 & 7,00 \\
\hline Cornella de LI. & 6,00 & 5,67 & 8,33 & 8,33 & 5,67 & 6,33 & 6,33 & 7,67 & 8,00 & 7,00 & 7,00 \\
\hline Esplugues de U. & 6,00 & 5,67 & 8,33 & 8,33 & 5,67 & 6,33 & 5,67 & 7,67 & 8,00 & 6,67 & 6,67 \\
\hline Sant Cugat delV. & 5,67 & 5,33 & 8,33 & 8,67 & 7,00 & 6,33 & 3,67 & 7,00 & 8,00 & 7,00 & 7,00 \\
\hline Badalona & 5,67 & 5,33 & 8,00 & 8,00 & 5,00 & 6,00 & 6,33 & 6,33 & 7,33 & 6,33 & 6,67 \\
\hline
\end{tabular}

Fuente: Elaboración propia

A partir de dichos promedios se realiza primeramente un análisis por columnas, quiere decirse por factores, se revisa primeramente el área central de negocios, centro de ciudad y nuevas áreas de negocio, mostrándose en el último renglón el promedio.

La tabla 9 muestra los resultados obtenidos, en donde los factores acceso a parada de autobús y acceso a grandes infraestructuras de transporte tienen importancia media, el resto de factores tienen alta importancia (rango de 8.44 a 9.33).

Tabla 9. Media aritmética de los factores para zonas centrales (etapa dos)

\begin{tabular}{|c|c|c|c|c|c|c|c|c|c|c|c|}
\hline & \multicolumn{9}{|c|}{ FACTORES } \\
\hline & 3 & 3 & 3 & 3 & 3 & 3,33 & 3,5 & 3,33 & 3,5 & 3,5 & 3,33 \\
\hline Zona & $\begin{array}{l}\text { Acceso a } \\
\text { parada de } \\
\text { autobús }\end{array}$ & $\begin{array}{l}\text { Acceso a los } \\
\text { consum idores } \\
\text { (clientes) }\end{array}$ & $\begin{array}{l}\text { Calidad de } \\
\text { urban ización }\end{array}$ & $\begin{array}{l}\text { Estado de } \\
\text { conservación } \\
\text { del stock } \\
\text { edificado }\end{array}$ & $\begin{array}{l}\text { Nivel de } \\
\text { ingresos }\end{array}$ & $\begin{array}{l}\text { Acceso a } \\
\text { avenidas } \\
\text { principales }\end{array}$ & $\begin{array}{l}\text { Acce so a } \\
\text { estación } \\
\text { de metro }\end{array}$ & $\begin{array}{l}\text { Acceso a } \\
\text { grandes } \\
\text { infraestructur } \\
\text { as del } \\
\text { transporte }\end{array}$ & $\begin{array}{l}\text { Calidad } \\
\text { del stock } \\
\text { edificado }\end{array}$ & $\begin{array}{l}\text { Compacidad } \\
\text { económ ico- } \\
\text { empresarial }\end{array}$ \\
\begin{tabular}{c|c|c|c|c|c|c|c|c|c|} 
Intensidad de \\
utilización del \\
stock \\
edificado
\end{tabular} \\
\hline Area Central de Negocios & 5,33 & 9,67 & 9,33 & 8,67 & 9,00 & 9,33 & 8,67 & 5,67 & 8,67 & 9,67 & 10,00 \\
\hline Centro Ciudad & 5,67 & 9,33 & 8,67 & 8,00 & 8,33 & 8,67 & 9,00 & 6,33 & 8,00 & 9,33 & 9,00 \\
\hline Nuevas Areas de Negocios & 5,00 & 7,33 & 9,00 & 9,00 & 8,33 & 8,33 & 8,33 & 5,67 & 8,67 & 9,00 & 8,33 \\
\hline Media Aritmética & 5,33 & 8,78 & 9,00 & 8,56 & 8,56 & 8,78 & 8,67 & 5,89 & 8,44 & 9,33 & 9,11 \\
\hline
\end{tabular}

Fuente: Elaboración propia

En un segundo paso se realiza el mismo procedimiento para la periferia, la tabla 10 muestra también los resultados obtenidos, destacando cuatro factores con importancia media, y el resto con puntaciones más altas (rango de 6.27 a 8.33), pero a su vez inferiores a los de las áreas centrales. 
Tabla 10. Media aritmética de los factores para zonas periféricas (etapa dos)

\begin{tabular}{|c|c|c|c|c|c|c|c|c|c|c|c|}
\hline & \multicolumn{9}{|c|}{ FACTORES } \\
\hline & 3 & 3 & 3 & 3 & 3 & 3,33 & 3,5 & 3,33 & 3,5 & 3,5 & 3,33 \\
\hline Zona & $\begin{array}{l}\text { Acceso a } \\
\text { parada de } \\
\text { autobús }\end{array}$ & $\begin{array}{l}\text { Acceso a los } \\
\text { consumidores } \\
\text { (clientes) }\end{array}$ & $\begin{array}{l}\text { Calidad de } \\
\text { urban ización }\end{array}$ & $\begin{array}{l}\text { Estado de } \\
\text { conservación } \\
\text { del stock } \\
\text { edificado }\end{array}$ & $\begin{array}{l}\text { Nivel de } \\
\text { ingresos }\end{array}$ & $\begin{array}{l}\text { Acceso a } \\
\text { avenidas } \\
\text { principales }\end{array}$ & $\begin{array}{l}\text { Acceso a } \\
\text { estación } \\
\text { de metro }\end{array}$ & $\begin{array}{l}\text { Acceso a } \\
\text { grandes } \\
\text { infraestructur } \\
\text { as del } \\
\text { tran sporte }\end{array}$ & $\begin{array}{l}\text { Calidad } \\
\text { del stock } \\
\text { edificado }\end{array}$ & $\begin{array}{l}\text { Compacidad } \\
\text { económ ico- } \\
\text { empresarial }\end{array}$ & $\begin{array}{l}\text { Intensidad de } \\
\text { utilización del } \\
\text { stock } \\
\text { edificado }\end{array}$ \\
\hline El Prat de Ll. & 6,00 & 5,67 & 8,33 & 8,33 & 6,00 & 6,33 & 6,33 & 8,00 & 8,33 & 7,00 & 7,00 \\
\hline Cornella de L. & 6,00 & 5,67 & 8,33 & 8,33 & 5,67 & 6,33 & 6,33 & 7,67 & 8,00 & 7,00 & 7,00 \\
\hline Esplugues de LI. & 6,00 & 5,67 & 8,33 & 8,33 & 5,67 & 6,33 & 5,67 & 7,67 & 8,00 & 6,67 & 6,67 \\
\hline Sant Cugat del V. & 5,67 & 5,33 & 8,33 & 8,67 & 7,00 & 6,33 & 3,67 & 7,00 & 8,00 & 7,00 & 7,00 \\
\hline Badalona & 5,67 & 5,33 & 8,00 & 8,00 & 5,00 & 6,00 & 6,33 & 6,33 & 7,33 & 6,33 & 6,67 \\
\hline Media Aritmética & 5,87 & 5,53 & 8,27 & 8,33 & 5,87 & 6,27 & 5,67 & 7,33 & 7,93 & 6,80 & 6,87 \\
\hline
\end{tabular}

Fuente: Elaboración propia

Retomando los promedios de la tabla 8, ahora se realiza un análisis por renglones, quiere decirse por zonas de valor y la correspondiente influencia de los diversos factores considerados; se obtiene una media aritmética ponderada por las diversas evaluaciones a los factores de la etapa uno.

La tabla 11 muestra los promedios ponderados para todas las zonas inmobiliarias, es de observarse que el Área Central de Negocios es la que tiene el promedio ponderado más alto, siguiendo el Centro de Ciudad y las Nuevas Áreas de Negocios; dichas zonas tienen promedio ponderado más alto (rango de 7,93 a 8,56) que la periferia (rango de 6,46 a 7,04).

\subsubsection{Resultados para la etapa dos de la consulta a expertos}

Para el Área Central de Negocios, Centro de Ciudad, y Nuevas Áreas de Negocios, los factores, acceso a parada de autobús y acceso a grandes infraestructuras de transporte tienen importancia media, el resto de los factores considerados tiene alta importancia.

Tabla 11. Media aritmética ponderada de factores para zonas inmobiliarias (etapa dos)

\begin{tabular}{|c|c|c|c|c|c|c|c|c|c|c|c|c|}
\hline & \multicolumn{11}{|c|}{ FACTORES } & \\
\hline & 3 & 3 & 3 & 3 & 3 & 3,33 & 3,5 & 3,33 & 3,5 & 3,5 & 3,33 & \\
\hline Zona & $\begin{array}{c}\text { Acceso a } \\
\text { parada de } \\
\text { autobús }\end{array}$ & $\begin{array}{c}\text { Acceso a los } \\
\text { consumidores } \\
\text { (cllentes) }\end{array}$ & $\begin{array}{c}\text { Calldad de } \\
\text { urbanización }\end{array}$ & $\begin{array}{l}\text { Estado de } \\
\text { conservadón } \\
\text { del stodk } \\
\text { edificado }\end{array}$ & $\begin{array}{l}\text { Nivel de } \\
\text { Ingresos }\end{array}$ & $\begin{array}{c}\text { Acceso a } \\
\text { avenidas } \\
\text { principales }\end{array}$ & $\begin{array}{l}\text { Acceso a } \\
\text { estación } \\
\text { de metro }\end{array}$ & \begin{tabular}{|c|} 
Acceso a \\
grandes \\
Infraestructur \\
as del \\
transporte
\end{tabular} & $\begin{array}{c}\text { Calldad } \\
\text { del stock } \\
\text { edificado }\end{array}$ & $\begin{array}{l}\text { Compacidad } \\
\text { económico- } \\
\text { empresarial }\end{array}$ & $\begin{array}{c}\text { Intensidad de } \\
\text { utillización del } \\
\text { stodok } \\
\text { edificado }\end{array}$ & $\begin{array}{l}\text { Promedlo } \\
\text { Ponderado }\end{array}$ \\
\hline Area Central de Negodos & 5,33 & 9,67 & 9,33 & 8,67 & 9,00 & 9,33 & 8,67 & 5,67 & 8,67 & 9,67 & 10,00 & 8,56 \\
\hline Centro Cludad & 5,67 & 9,33 & 8,67 & 8,00 & 8,33 & 8,67 & 9,00 & 6,33 & 8,00 & 9,33 & 9,00 & 8,23 \\
\hline Nuevas Areas de Negocios & 5,00 & 7,33 & 9,00 & 9,00 & 8,33 & 8,33 & 8,33 & 5,67 & 8,67 & 9,00 & 8,33 & 7,93 \\
\hline El Prat de Ll. & 6,00 & 5,67 & 8,33 & 8,33 & 6,00 & 6,33 & 6,33 & 8,00 & 8,33 & 7,00 & 7,00 & 7,04 \\
\hline Cornella de U. & 6,00 & 5,67 & 8,33 & 8,33 & 5,67 & 6,33 & 6,33 & 7,67 & 8,00 & 7,00 & 7,00 & 6,95 \\
\hline Esplugues de LI. & 6,00 & 5,67 & 8,33 & 8,33 & 5,67 & 6,33 & 5,67 & 7,67 & 8,00 & 6,67 & 6,67 & 6,82 \\
\hline Sant Cugat del V. & 5,67 & 5,33 & 8,33 & 8,67 & 7,00 & 6,33 & 3,67 & 7,00 & 8,00 & 7,00 & 7,00 & 6,71 \\
\hline Badalona & 5,67 & 5,33 & 8,00 & 8,00 & 5,00 & 6,00 & 6,33 & 6,33 & 7,33 & 6,33 & 6,67 & 6,46 \\
\hline
\end{tabular}

Fuente: Elaboración propia

Para la Periferia, los factores, acceso a parada de autobús, acceso a consumidores, nivel de ingresos y acceso a estación de metro, tienen importancia media, el resto de los factores considerados tiene mayor importancia.

El Área Central de Negocios es la que tiene el promedio ponderado de influencia de los diversos factores considerados, más alto, siguiendo el Centro de Ciudad y las Nuevas Áreas de Negocios; dichas zonas tienen un promedio ponderado de influencia más alto (rango de 7,93 a 8,56) que la periferia (rango de 6,46 a 7,04), confirmándose así los niveles y la importancia de los factores considerados para cada zona de valor inmobiliario. 


\section{Conclusiones}

Indudablemente, la consulta a expertos puede ser un importante recurso en la investigación, para el presente trabajo no es la excepción. Sin embargo, se debe señalar que los resultados obtenidos para la primera etapa se ven con menos reserva que los obtenidos en la segunda, principalmente por el número de expertos que participaron en cada una de ellas.

Hay aspectos dentro del proceso de la consulta a expertos, que son de difícil control para el investigador, como lo es por ejemplo la dificultad de la tarea, por lo que se debe intentar ser lo menos ambiguos posible. Otros aspectos que se consideran fundamentales en el método se refieren, como se menciona anteriormente, al número de expertos y su selección.

También es importante mencionar la recomendación (Escobar y Cuervo, 2008), de que el investigador debe propiciar el contexto adecuado para obtener la mayor cantidad de información posible de los expertos y pedir opiniones adicionales sobre la prueba que pueden dar información sobre aspectos no evaluados en la consulta.

\section{Bibliografía}

ASTIGARRAGA, E. El método Delphi. San Sebastian. Universidad Deusto. Disponible en: http://www.prospectiva.eu/zaharra/Metodo_delphi.pdf, 2008. 14 p.

CRUZ, M.; Campano, A. El procesamiento de la información en investigaciones educacionales. La Habana: Education Cabana; 2008.

GARCÍA, V. M.; Suárez, M. M. El método Delphi para la consulta a expertos en la investigación científica. En: Revista Cubana de Salud Pública, 39 (2), 253-267. 2013.

HYRKAS, K., Appelqvist-Schmidlechner, K.; Oksa, L. Validating an instrument for clinical supervision using an expert panel. En: International Journal of nursing studies, 40 (6), 619-625. 2003.

JONES, J.; Hunter, D. Qualitative Research: Consensus methods for medical and health services research. En: BMJ, 311-376. 1995.

LANDETA, Jon. El método Delphi. Una técnica de previsión para la incertidumbre. Ariel. Barcelona, España. 1999.

LINSTONE, H., Turoff, M. The Delphi Method. Techniques and Applications. Addison-Wesley, 1975, p 3.

MC GARTLAND, D.; Berg, M.; Tebb, S. S.; Lee, E. S. \& Rauch, S. Objectifying content validity: Conducting a content validity study in social work research. En: Social Work Research, 27 (2), 94-104. 2003.

SILVESTRE O. J. R. Rentabilidad de los inmuebles de oficinas en el Área Metropolitana de Barcelona. Tesis doctoral (inédita), Universidad Politécnica de Cataluña. 2017.

SKJONG, R. \& Wentworth, B. Expert Judgement and risk perception. Fecha de consulta: 15 de enero de 2006. Disponible en: http://research.dnv.com/skj/Papers/SkjWen.pdf. 2000.

Citación: SILVESTRE ORTIZ, J. R. La consulta a expertos, un importante recurso en la investigación. En: Libro de proceedings, CTV 2018. XII Congreso Internacional Ciudad y Territorio Virtual. "Ciudades y Territorios Inteligentes". UNCuyo, Mendoza, 5-7 septiembre 2018. Barcelona: CPSV, 2018, p. 181-192. 